\title{
Strength of reduced two-body spin-orbit interaction from chiral three-nucleon force
}

\author{
M. Kohno \\ Physics Division, Kyushu Dental College, Kitakyushu 803-8580, Japan
}

\begin{abstract}
The contribution of a chiral three-nucleon force to the strength of an effective spin-orbit coupling is estimated. We first construct a reduced two-body interaction by folding one-nucleon degrees of freedom of the three-nucleon force in nuclear matter. The spin-orbit strength is evaluated by a Scheerbaum factor obtained by the $G$-matrix calculation in nuclear matter with the two-nucleon interaction plus the reduced two-nucleon interaction. The problem of the insufficiency of modern realistic two-nucleon interactions to account for the empirical spin-orbit strength is resolved. It is also indicated that the spin-orbit coupling is weaker in the neutron-rich environment. Because the spin-orbit component from the three-nucleon force is determined by the low-energy constants fixed in the two-nucleon sector, there is little uncertainty in the present estimation.
\end{abstract}

PACS numbers: 21.30.Fe, 21.45.Ff, 21.65.-f

Spin-orbit field in atomic nuclei is essential to reproduce well-established single-particle shell structure. The empirical strength of the spin-orbit potential, however, has not been fully understood on the basis of the realistic nucleon-nucleon force. The possible role of intermediate isobar $\Delta$-excitation to the nuclear spin-orbit field was considered in parallel with the construction of the twopion-exchange three-nucleon force (3NF) by Fujita and Miyazawa 1]. The problem was reinvestigated in the early 1980s [2, 3] to search for the additional spin-orbit strength. Later, the Illinois group showed [4] that their $3 \mathrm{NF}$ makes a substantial contribution to the spin-orbit splitting in ${ }^{15} \mathrm{~N}$.

Kaiser and his collaborators investigated, in their several papers [5 7], the nuclear spin-orbit coupling in the framework of chiral perturbation theory. The large contributions generated by iterated one-pion exchange and the $3 \mathrm{NF}$ almost cancel each other [5, [6], and the shortrange spin-orbit strength in the form of the effective fournucleon contact-coupling deduced from realistic nucleonnucleon interactions accounts well [7] for the empirical one. Because the contact-interaction in the chiral perturbation, however, is still needed to be regulated for the application to low-energy nuclear structure calculations, and their arguments for various contributions seem not to be fully unified, it is worthwhile to analyze the effective strength of the spin-orbit coupling by applying the established microscopic theory, namely the lowest-order Brueckner theory, to the two-nucleon and three-nucleon interactions in the chiral effective field theory (Ch-EFT).

The Thomas form of an average single-particle spinorbit potential has been used to describe nucleon spinorbit coupling:

$$
U_{\ell s}^{0} \frac{1}{r} \frac{d \rho(r)}{d r} \ell \cdot \sigma
$$

where the radial function $\rho(r)$ is a nucleon total density distribution. The relation of the strength $U_{\ell s}^{0}$ to a two-body effective spin-orbit interaction was derived by Scheerbaum [8]. By defining the constant $B_{S}(\bar{q})$ for the triplet odd component of the effective two-body spin- orbit interaction $v_{\ell s}^{3 O}(r)$

$$
B_{S}(\bar{q})=-\frac{2 \pi}{\bar{q}} \int_{0}^{\infty} d r r^{3} j_{1}(\bar{q} r) v_{\ell s}^{3 O}(r)
$$

with $j_{1}$ being a spherical Bessel function, the singleparticle spin-orbit potential for spin-saturated nuclei may be written as

$$
U_{\ell s, \tau}(r)=\frac{1}{2} B_{S}(\bar{q}) \frac{1}{r} \frac{d\left\{\rho(r)+\rho_{\tau}(r)\right\}}{d r} \ell \cdot \boldsymbol{\sigma},
$$

where $\tau$ specifies either a proton or neutron. We refer to $B_{S}(\bar{q})$ as a Scheerbaum factor, which is different from the original constant in Ref. [8] by a factor of $-\frac{2 \pi}{3}$. Scheerbaum prescribed $\bar{q} \approx 0.7 \mathrm{fm}^{-1}$ on the basis of the wavelength of the density distribution. We employ this prescription. If we assume a naive relation $\rho_{p}(r)=\rho_{n}(r)=\frac{1}{2} \rho(r)$, we recover the Thomas form, Eq. (11), with $U_{\ell s}^{0}=\frac{3}{4} B_{S}(\bar{q})$. It has also been customary to use a $\delta$-type two-body spin-orbit interaction

$$
i W\left(\boldsymbol{\sigma}_{1}+\boldsymbol{\sigma}_{2}\right) \cdot\left(\nabla_{r} \times \delta(\boldsymbol{r}) \nabla_{r}\right)
$$

in nuclear Hartree-Fock calculations using $\delta$-type Skyrme interactions [9, 10] and even with finite range effective forces, e.g., the Gogny force [11]. This two-body force provides a single-particle spin-orbit potential:

$$
\frac{1}{2} W \frac{1}{r} \frac{d\left\{\rho(r)+\rho_{\tau}(r)\right\}}{d r} \ell \cdot \boldsymbol{\sigma} .
$$

Therefore, the strength $W$ may be identified as the Scheerbaum factor $B_{S}(\bar{q})$. The empirical value of $W$ is around $120 \mathrm{MeV} \cdot \mathrm{fm}^{5}$ in various nuclear HartreeFock calculations. As will be shown below, the modern nucleon-nucleon interactions underestimate the spinorbit strength by about $25 \%$.

Applying Scheerbaum's formulation to the momentum-space $G$-matrix calculation in nuclear matter with the Fermi momentum $k_{F}$, we obtain the 
corresponding spin-orbit strength as follows [12]:

$$
\begin{array}{r}
B_{S}(\bar{q})=\frac{1}{k_{F}^{3}} \sum_{J T}(2 J+1)(2 T+1) \int_{0}^{q_{\max }} d q \\
\times W(\bar{q}, q)\left\{(J+2) G_{1 J+1,1 J+1}^{J T}(q)+G_{1 J, 1 J}^{J T}(q)\right. \\
\left.-(J-1) G_{1 J-1,1 J-1}^{J T}(q)\right\} .
\end{array}
$$

Here, $q_{\max }=\frac{1}{2}\left(k_{F}+\bar{q}\right)$ and the weight factor $W(\bar{q}, q)$ is

$$
W(\bar{q}, q)=\left\{\begin{array}{l}
\theta\left(k_{F}-\bar{q}\right) \text { for } 0 \leq q \leq \frac{\left|k_{F}-\bar{q}\right|}{2} \\
\frac{k_{F}^{2}-(\bar{q}-2 q)^{2}}{8 \bar{q} q} \text { for } \frac{\left|k_{F}-\bar{q}\right|}{2} \leq q \leq \frac{k_{F}+\bar{q}}{2}
\end{array}\right.
$$

where $\theta\left(k_{F}-\bar{q}\right)$ is a step function. In Eq. (6), $G_{1 \ell^{\prime}, 1 \ell}^{J T}$ is the abbreviation of the momentum-space diagonal $G$ matrix element in the spin-triplet channel with the total isospin $T$, total spin $J$, and orbital momenta $\ell^{\prime}$ and $\ell$.

Calculating $B_{S}(\bar{q})$ in the lowest-order Brueckner theory with the continuous prescription for intermediate spectra, as presented below explicitly in Table 1, modern two-body nucleon-nucleon potentials are found to give smaller values of around $90 \mathrm{Mev} \cdot \mathrm{fm}^{5}$ compared with the empirical one. As has been well known that LOBT calculations in symmetric nuclear matter with realistic twonucleon force do not reproduce correct saturation property. However, in most case, calculated energies at the empirical saturation point $k_{F}=1.35 \mathrm{fm}^{-1}$ are close to the empirical energy of about $-16 \mathrm{MeV}$. This suggests that $G$ matrices provide basic information on the effective nucleon-nucleon interaction in the nuclear medium, by incorporating important short-range correlations, Pauli effects and dispersion effects.

Now we consider the contribution of the 3NF. In this article, we estimate it in a two-step procedure. First, the $3 \mathrm{NF} v_{123}$ defined in momentum space is reduced to an effective two-nucleon interaction $v_{12(3)}$ by folding onenucleon degrees of freedom:

$$
\left\langle\boldsymbol{k}_{1}^{\prime} \sigma_{1}^{\prime} \tau_{1}^{\prime}, \boldsymbol{k}_{2}^{\prime} \sigma_{2}^{\prime} \tau_{2}^{\prime}\left|v_{12(3)}\right| \boldsymbol{k}_{1} \sigma_{1} \tau_{1}, \boldsymbol{k}_{2} \sigma_{2} \tau_{2}\right\rangle_{A}=\frac{1}{3} \sum_{\boldsymbol{k}_{3} \sigma_{3} \tau_{3}}\left\langle\boldsymbol{k}_{1}^{\prime} \sigma_{1}^{\prime} \tau_{1}^{\prime}, \boldsymbol{k}_{2}^{\prime} \sigma_{2}^{\prime} \tau_{2}^{\prime}, \boldsymbol{k}_{3} \sigma_{3} \tau_{3}\left|v_{123}\right| \boldsymbol{k}_{1} \sigma_{1} \tau_{1}, \boldsymbol{k}_{2} \sigma_{2} \tau_{2}, \boldsymbol{k}_{3} \sigma_{3} \tau_{3}\right\rangle_{A}
$$

Here, we have to assume that remaining two nucleons are in the center-of-mass frame, namely $\boldsymbol{k}_{1}^{\prime}+\boldsymbol{k}_{2}^{\prime}=\boldsymbol{k}_{1}+\boldsymbol{k}_{2}=$ 0 . The density-dependent effective two-nucleon interaction as the effect of the 3NF has been commonly introduced in the literature 13 15]. Note that the suffix $A$ means an antisymmetrized matrix element; namely $|a b\rangle_{A} \equiv|a b-b a\rangle$ and $|a b c\rangle_{A} \equiv \mid a b c-a c b+b c a-b a c+c a b-$ $c b a\rangle$, and the factor $\frac{1}{3}$ in Eq. (8) is an additional statistical one. This statistical factor has been often slipped in the literature. The recent derivation of the effective twobody interaction from the Ch-EFT 3NF by Holt, Kaiser and Weise [15] also seems not to be an exception. If an adjustable strength is introduced, the statistical factor may be hidden in the fitting procedure. In our case of using the Ch-EFT 3NF, the low-energy constants except for $c_{D}$ and $c_{E}$ are fixed. Although there may be a room to adjust $c_{D}$ and $c_{E}$, the contributions to the energy from these terms are rather small, if they are in a reasonable range. In addition, $c_{D}$ and $c_{E}$ do not contribute to the reduce two-nucleon spin-orbit interaction. By comparing the nuclear matter energy directly calculated from $v_{123}$ and that by the reduced $v_{12(3)}$, the error due to this approximation can be checked to be less than $10 \%$, if we calculate Born energy without including a form factor.

To explain the procedure of obtaining $v_{12(3)}$ more explicitly, we write the reduced spin-orbit component orig- inating from the $c_{1}$ term of the Ch-EFT $3 \mathrm{NF}$ :

$$
-\frac{c_{1} g_{A}^{2} m_{\pi}^{2}}{f_{\pi}^{4}} \sum_{1 \leq i<j \leq 3} \frac{\left(\boldsymbol{\sigma}_{i} \cdot \boldsymbol{q}_{i}\right)\left(\boldsymbol{\sigma}_{j} \cdot \boldsymbol{q}_{j}\right)}{\left(\boldsymbol{q}_{i}^{2}+m_{\pi}^{2}\right)\left(\boldsymbol{q}_{j}^{2}+m_{\pi}^{2}\right)}\left(\boldsymbol{\tau}_{i} \cdot \boldsymbol{\tau}_{j}\right)
$$

where $g_{A}=1.29, f_{\pi}=92.4 \mathrm{MeV}, m_{\pi}$ is a pion mass, and $\boldsymbol{q}_{i}$ is a momentum transfer of the $i$-th nucleon. The momentum transfer of the third nucleon $k$ is dictated by the relation $\boldsymbol{q}_{k}=-\boldsymbol{q}_{i}-\boldsymbol{q}_{j}$. The folding of the $3 \mathrm{NF}$ by one nucleon is carried out without incorporating a threebody form factor. A form factor is later introduced on the two-body level. The folding in symmetric nuclear matter with the Fermi momentum $k_{F}$ gives, besides the central and tensor components, the following spin-orbit term:

$$
\begin{aligned}
& \frac{c_{1} g_{A}^{2} m_{\pi}^{2}}{f_{\pi}^{4}} \frac{1}{(2 \pi)^{3}} \iiint_{\left|\boldsymbol{k}_{3}\right| \leq k_{F}} d \boldsymbol{k}_{3} \\
& \times \frac{i\left(\boldsymbol{\sigma}_{1}+\boldsymbol{\sigma}_{2}\right) \cdot\left(-\boldsymbol{k}_{1}^{\prime} \times \boldsymbol{k}_{1}+\left(\boldsymbol{k}_{1}^{\prime}-\boldsymbol{k}_{1}\right) \times \boldsymbol{k}_{3}\right)}{\left(\left(\boldsymbol{k}_{1}^{\prime}-\boldsymbol{k}_{3}\right)^{2}+m_{\pi}^{2}\right)\left(\left(\boldsymbol{k}_{1}-\boldsymbol{k}_{3}\right)^{2}+m_{\pi}^{2}\right)} .
\end{aligned}
$$

When carrying out the folding in pure neutron matter, the restriction of the isotopic spin brings about an additional factor of $\frac{1}{3}$.

The partial-wave decomposition of the above spin-orbit term becomes

$$
\begin{aligned}
& -\delta_{S 1} \frac{c_{1} g_{A}^{2} m_{\pi}^{2}}{f_{\pi}^{4}} \frac{\ell(\ell+1)+2-J(J+1)}{2 \ell+1} \\
& \left\{Q_{W, 0}^{\ell-1}\left(k_{1}^{\prime}, k_{1}\right)-Q_{W, 0}^{\ell+1}\left(k_{1}^{\prime}, k_{1}\right)-W_{\ell s, 0}^{\ell}\left(k_{1}^{\prime}, k_{1}\right)\right\}(11)
\end{aligned}
$$


for the orbital and total angular momenta $\ell$ and $J$. The functions $Q_{W, 0}^{\ell}$ and $W_{\ell s, 0}^{\ell}$ are defined by

$$
\begin{aligned}
Q_{W, 0}^{\ell}\left(k_{1}^{\prime}, k_{1}\right) \equiv & \frac{2 \pi}{(2 \pi)^{3}} \frac{1}{2} \int_{0}^{k_{F}} d k_{3} Q_{\ell}\left(x^{\prime}\right) Q_{\ell}(x) \\
W_{\ell s, 0}^{\ell}\left(k_{1}^{\prime}, k_{1}\right) \equiv & \frac{2 \pi}{(2 \pi)^{3}} \frac{1}{2 k_{1}^{\prime} k_{1}} \int_{0}^{k_{F}} d k_{3} k_{3} \\
& \times\left\{k_{1}^{\prime} Q_{\ell}(x)\left(Q_{\ell-1}\left(x^{\prime}\right)-Q_{\ell+1}\left(x^{\prime}\right)\right)\right. \\
& \left.+k_{1} Q_{\ell}\left(x^{\prime}\right)\left(Q_{\ell-1}(x)-Q_{\ell+1}(x)\right)\right\}
\end{aligned}
$$

where $Q_{\ell}(x)$ is a Legendre function of the second kind, and $x^{\prime} \equiv \frac{k_{3}^{2}+k_{1}^{\prime 2}+m_{\pi}^{2}}{2 k_{1}^{\prime} k_{3}}$ and $x \equiv \frac{k_{3}^{2}+k_{1}^{2}+m_{\pi}^{2}}{2 k_{1} k_{3}}$, respectively.

The spin-orbit component arises also from the $c_{3}$ term of the Ch-EFT 3NF. This case, in addition to the replacement of the coupling constant, an additional factor $\left(\boldsymbol{k}_{1}^{\prime}-\boldsymbol{k}_{3}\right) \cdot\left(\boldsymbol{k}_{3}-\boldsymbol{k}_{1}\right)$ appears in the denominator in Eq. (10). The partial-wave decomposition reads

$$
\begin{array}{r}
\delta_{S 1} \frac{c_{3} g_{A}^{2}}{2 f_{\pi}^{4}} \frac{\ell(\ell+1)+2-J(J+1)}{2 \ell+1}\left[\left(m_{\pi}^{2}+\frac{1}{2}\left(k_{1}^{\prime 2}+k_{1}^{2}\right)\right)\left\{Q_{W, 0}^{\ell-1}\left(k_{1}^{\prime}, k_{1}\right)-Q_{W, 0}^{\ell+1}\left(k_{1}^{\prime}, k_{1}\right)-W_{\ell s, 0}^{\ell}\left(k_{1}^{\prime}, k_{1}\right)\right\}\right. \\
+3 k_{1}^{\prime} k_{1}\left\{Q_{W, 0}^{\ell}\left(k_{1}^{\prime}, k_{1}\right)-(\ell-1) Q_{W, 0}^{\ell-2}\left(k_{1}^{\prime}, k_{1}\right)+(\ell+2) Q_{W, 0}^{\ell+2}\left(k_{1}^{\prime}, k_{1}\right)+\frac{\ell-1}{2 \ell-1} W_{\ell s, 0}^{\ell-1}\left(k_{1}^{\prime}, k_{1}\right)+\frac{\ell+2}{2 \ell+3} W_{\ell s, 0}^{\ell+1}\left(k_{1}^{\prime}, k_{1}\right)\right\} \\
\left.-\delta_{\ell 1} \frac{k_{1}^{\prime} k_{1}}{2}\left(F_{0}\left(k_{1}^{\prime}\right)+F_{0}\left(k_{1}\right)-F_{1}\left(k_{1}^{\prime}\right)-F_{1}\left(k_{1}\right)\right)\right]
\end{array}
$$

where the new functions $F_{0}(k)$ and $F_{1}(k)$ are defined by

$$
\begin{gathered}
F_{0}(k) \equiv \frac{1}{(2 \pi)^{3}} \iiint_{\left|\boldsymbol{k}_{3}\right| \leq k_{F}} d \boldsymbol{k}_{3} \frac{1}{\left(\boldsymbol{k}-\boldsymbol{k}_{3}\right)^{2}+m_{\pi}^{2}}, \\
F_{1}(k) \equiv \frac{1}{(2 \pi)^{3}} \frac{1}{k^{2}} \iiint_{\left|\boldsymbol{k}_{3}\right| \leq k_{F}} d \boldsymbol{k}_{3} \frac{\boldsymbol{k} \cdot \boldsymbol{k}_{3}}{\left(\boldsymbol{k}-\boldsymbol{k}_{3}\right)^{2}+m_{\pi}^{2}} .
\end{gathered}
$$

Adding the reduced two-nucleon interaction to the ChEFT two-nucleon interaction, we repeat the LOBT $G$ matrix calculation. Although explicit expressions are not shown in this Letter except for the spin-orbit part, we include all central, tensor and spin-orbit components of the reduced interaction $v_{12(3)}$. The form factor in a functional form of $f\left(k_{1}^{\prime}, k_{1}\right)=\exp \left\{-\left[\left(k_{1}^{\prime} / \Lambda\right)^{4}+\left(k_{1} / \Lambda\right)^{4}\right]\right\}$ is introduced for $v_{12(3)}$ with the cut-off mass $\Lambda=550 \mathrm{MeV}$. We use the low-energy constants fixed for the Jülich ChEFT potential by Hebeler et al. [16]; $c_{D}=-4.381$, and $c_{E}=-1.126$. Other constants are $c_{1}=-0.81 \mathrm{GeV}^{-1}$, $c_{3}=-3.4 \mathrm{GeV}^{-1}$, and $c_{4}=3.4 \mathrm{GeV}^{-1}$. Because the reduction of the $3 \mathrm{NF}$ to the two-nucleon force was carried out in nuclear matter, $v_{12(3)}$ may not be directly applied to very light nuclei, such as ${ }^{3} \mathrm{H}$ and ${ }^{4} \mathrm{He}$.

First, we comment on calculated saturation curves, which are given in Fig. 1. Without the contribution of the $3 \mathrm{NF}$, the saturation curve attains its minimum at larger $k_{F}$ as a function of the Fermi momentum $k_{F}$ than the empirical saturation momentum, as has been known. Nucleon-nucleon interactions, AV18 [17], NSC97 18], and Jülich $\mathrm{N}^{3} \mathrm{LO}$ with the cutoff mass of $550 \mathrm{MeV}$ 19] give similar saturation curves, and the CD-Bonn potential [20] predicts somewhat deeper binding. For the reference of what saturation curve is preferable for nuclear mean filed calculations, we also show the result with the Gogny D1S interaction [1].

\begin{tabular}{crrrrr}
\hline \hline$k_{F}=1.35 \mathrm{fm}^{-1}$ & AV18 & NSC97 & CD-B N & LO N & \\
\hline \hline$B_{S}(T=0)$ & 2.09 & 1.9 & 3.1 & 2.5 & 7.0 \\
$B_{S}(T=1)$ & 86.4 & 86.7 & 90.2 & 84.6 & 116.2 \\
\hline$B_{S}(\bar{q})$ & 88.4 & 88.6 & 93.3 & 87.1 & 123.2 \\
\hline \hline$k_{F}=1.07 \mathrm{fm}^{-1}$ & AV18 & NSC97 & CD-B N N & $\mathrm{LO} \mathrm{N}^{3} \mathrm{LO}+3 \mathrm{NF}$ \\
\hline$B_{S}(T=0)$ & 1.4 & 1.3 & 2.3 & 1.6 & 4.1 \\
$B_{S}(T=1)$ & 88.1 & 88.7 & 92.2 & 86.5 & 106.7 \\
\hline$B_{S}(\bar{q})$ & 89.5 & 90.0 & 94.5 & 88.1 & 110.8 \\
\hline \hline
\end{tabular}

TABLE I: $B_{S}(\bar{q})$ in the unit of $\mathrm{MeV} \cdot \mathrm{fm}^{5}$ given by Eq. (6) with $\bar{q}=0.7 \mathrm{fm}^{-1}$ for modern nucleon-nucleon interaction: AV18 [17], NSC97 [18], CD-Bonn [20], and Jülich $\mathrm{N}^{3} \mathrm{LO}$ [19]. The last entry is the result with including the reduced two-body interaction from the Ch-EFT 3NF.

The thin dotted curve shows the result in which the plane wave expectation value of the $3 \mathrm{NF} v_{123}$ is added to the result of the two-nucleon $\mathrm{N}^{3} \mathrm{LO}$. The thick dotted curve alongside the thin dotted curve is the result with the plane wave expectation value of the reduced twonucleon interaction $v_{12(3)}$. The difference between the thin and thick curves is due to the difference of the formfactors and the necessary approximation $\boldsymbol{k}_{1}^{\prime}+\boldsymbol{k}_{2}^{\prime}=\boldsymbol{k}_{1}+$ $\boldsymbol{k}_{2}=0$ in Eq. (8).

The solid curve is the result of the $G$-matrix calculation with including the reduced two-nucleon interaction, $v_{12(3)}$. Although the energy is seen to be underestimated by a few $\mathrm{MeV}$, the saturation property is largely improved by the repulsive contribution from the threenucleon force. It is not necessary at present to expect a perfect agreement with the empirical properties in the LOBT calculation in nuclear matter. 


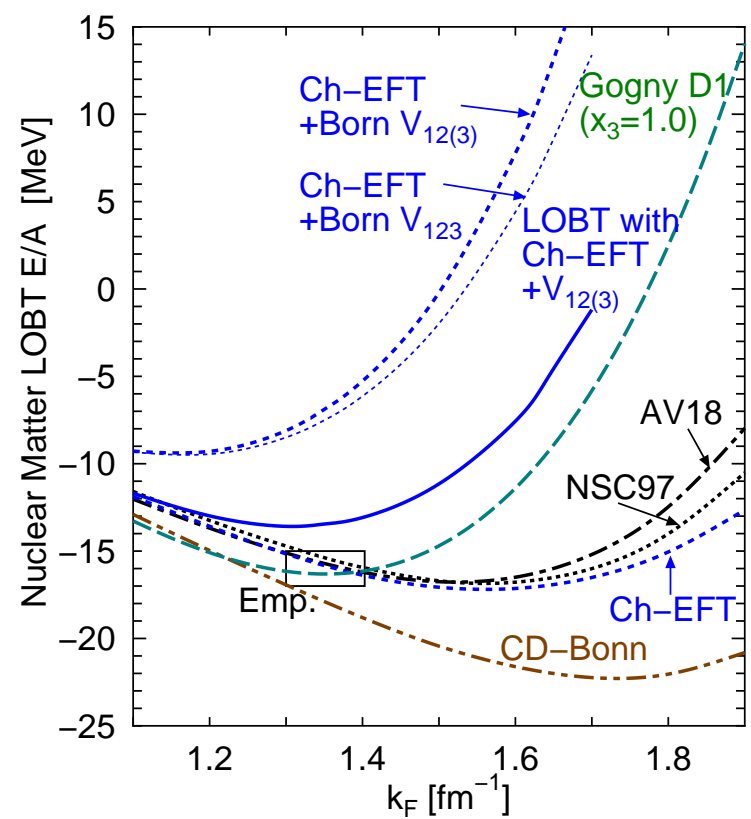

FIG. 1: Saturation curves in symmetric nuclear matter.

Now we examine the spin-orbit strength. We tabulate values for $B_{S}(\bar{q})$ of Eq. (6) at $\bar{q}=0.7 \mathrm{fm}^{-1}$ calculated in the LOBT with modern nucleon-nucleon interactions: AV18 [17], NSC97 [18], CD-Bonn [20], and Jülich $\mathrm{N}^{3} \mathrm{LO}$ [19]. The Scheerbaum factors obtained by realistic twonucleon forces are seen to be similar but insufficient to explain the strength needed in nuclear mean field calculations. Namely only about three-fourths of the empirically needed strength is accounted for. The two-body part of the Ch-EFT, $\mathrm{N}^{3} \mathrm{LO}$, shows little difference with other realistic two-nucleon force. It is also noticed that values at $k_{F}=1.07 \mathrm{fm}^{-1}$, namely at the half of the normal density, change little from those at the normal density with $k_{F}=1.35 \mathrm{fm}^{-1}$. It turns out, as the last column of Table I shows, that the addition of the reduced two-body interaction from the Ch-EFT 3NF bring about a good effect to fill the gap, though the $3 \mathrm{NF}$ contribution is smaller at $k_{F}=1.07 \mathrm{fm}^{-1}$. This is in accord with the important role of the $3 \mathrm{NF}$ to the spin-orbit splitting demonstrated in quantum Monte Carlo calculations of low-energy neutron-alpha scattering [21]. Although there are ambiguities from the form factor and uncertainties inherent in the folding procedure without taking into account nucleon-nucleon correlations, no additional adjustable parameter exists, because low-energy constants $c_{1}$ and $c_{3}$ which contribute solely to the spinorbit strength are determined on the two-nucleon sector.

As noted after Eq. (10), the reduced two-body spin- orbit term in neutron matter is one-third of that in symmetric nuclear matter. Actual $G$-matrix calculations using the Ch-EFT $\mathrm{N}^{3} \mathrm{LO}$ plus $v_{12(3)}$ in pure neutron matter with $k_{F}^{n}=1.35 \mathrm{fm}^{-1}$ tell that $B_{S}(\bar{q})$ values at $\bar{q}=0.7$ $\mathrm{fm}^{-1}$ are 84.7 and $93.5 \mathrm{MeV} \cdot \mathrm{fm}^{5}$ without and with the reduced two-nucleon interaction $v_{12(3)}$, respectively. If $k_{F}^{n}=1.07 \mathrm{fm}^{-1}$ is assumed, the corresponding values are 87.0 and $94.6 \mathrm{MeV} \cdot \mathrm{fm}^{5}$, respectively. Again, the $k_{F}^{n}$ dependence is weak. While the spin-orbit strength from the two-nucleon force is scarcely different from that in symmetric nuclear matter, the additional contribution from the three-nucleon force is in fact almost one-third of that in symmetric nuclear matter. Thus, the spin-orbit strength is expected to be smaller in the neutron-rich environment. This seems to be consistent with the trend observed in the shell structure near the neutron drip line 22] that a decreasing spin-orbit interaction is preferable with increasing neutron excess.

In summary, we have estimated quantitatively the contribution of the three-nucleon force of the chiral effective field theory to the single-particle spin-orbit strength, using the formulation by Scheerbaum [8]. We first introduced the reduced two-body interaction by folding onenucleon degrees of freedom of the $3 \mathrm{NF}$ in nuclear matter. Making partial-wave expansion of the resulting two-body interaction and adding it to the genuine two-nucleon interaction with including the necessary statistical factor of $\frac{1}{3}$, we carried out LOBT $G$-matrix calculations in infinite matter and evaluated the Scheerbaum factor corresponding to the spin-orbit strength. Because the spinorbit field in the atomic nuclei is fundamentally important as the nuclear magic numbers exhibit, it is important to learn that the inclusion of the $3 \mathrm{NF}$ in the chiral effective field theory can account for the spin-orbit strength empirically required for nuclear mean filed calculations. Because the relevant low-energy constants $c_{1}$ and $c_{3}$ are determined in the two-nucleon interaction sector, there should be little uncertainty for the additional spin-orbit strength except for the treatment of the twobody form factor. We have also noted that the additional spin-orbit strength from the $3 \mathrm{NF}$ should be weaker in neutron-excess nuclei.

\section{Acknowledgments}

This work is supported by Grant-in-Aid for Scientific Research (C) from the Japan Society for the Promotion of Science (Grant No. 22540288). The author thanks H. Kamada for valuable comments concerning the ChEFT interaction. He is also grateful to M. Yahiro for his interest in this work.
[1] J. Fujita and H. Miyazawa, Prog. Theor. Phys 17, 366 (1957).
[2] K. Ohta, T. Terasawa, and M. Tohyama, Phys. Rev. C 22, 2233 (1980) 
[3] K. Andō and H. Bandō, Prog. Theor. Phys 66, 227 (1981).

[4] S.C. Pieper and V.R. Pandharipande, Phys. Rev. Lett. 70, 2541 (1993).

[5] N. Kaiser, S. Fritsch, and W. Weise, Nucl. Phys. A724, 47 (2003).

[6] N. Kaiser, Phys. Rev. C68, 054001 (2003).

[7] N. Kaiser, Phys. Rev. C70, 034307 (2004).

[8] R.R. Scheerbaum, Nucl. Phys. A257, 77 (1976).

[9] M. Beiner, H. Flocard, N. Van Giai, and P. Quentin, Nucl. Phys. A238, 29 (1975).

[10] H. Krivine, J. Treiner, and O. Bohigas, Nucl. Phys. A336, 155 (1980).

[11] D. Gogny and R. Padjen, Nucl. Phys. 293, 365 (1977).

[12] Y. Fujiwara, M. Kohno, T. Fujita, C. Nakamoto, and Y. Suzuki, Nucl. Phys. A674, 493 (2000).

[13] T. Kasahara, Y. Akaishi, and H. Tanaka, Prog. Theor. Phys. Suppl. 56, 96 (1974).
[14] B. Friedman and V.R. Pandharipande, Nucl. Phys. A361, 502 (1981).

[15] J.W. Holt, N. Kaiser, and W. Weise, Phys. Rev. C 81, 024002 (2010).

[16] K. Hebeler, S.K. Bogner, R.J. Furnstahl, A. Nogga, and A. Schwenk, Phys. Rev. C83, 031301(R) (2011).

[17] R.B. Wiringa, V.G.J.Stoks, and R. Schiavilla, Phys. Rev. C51, 38 (1995).

[18] T. A. Rijken, V. G. J. Stoks, and Y. Yamamoto, Phys. Rev. C 59, 21 (1999).

[19] E. Epelbaum, W. Göckle, and U.-G. Meißner, Nucl. Phys. A747, 362 (2005).

[20] R. Machleidt, Phys. Rev. C63, 024001 (2001).

[21] K.M. Nollett, S.C. Pieper, R.B. Wiringa, J. Carlson, and G.M. Hale, Phys. Rev. Lett. 99, 022502 (2007).

[22] J.P. Schiffer et al., Phys. Rev. Lett. 92, 162501 (2004). 